\title{
Effect of process parameters on growth rate and diameter of nano-porous alumina templates
}

\author{
P CHOWDHURY* , K RAGHUVARAN, M KRISHNAN, HARISH C BARSHILIA And K S RAJAM \\ Surface Engineering Division, National Aerospace Laboratories (CSIR), Bangalore 560 017, India
}

MS received 29 July 2010; revised 4 October 2010

\begin{abstract}
Anodic aluminium oxide (AAO) template with hexagonal shaped nano-pores with high aspect ratio was fabricated by two-step anodization processes from high purity aluminium foil. It was observed that pore dimensions were affected by anodizing voltage, electrolyte temperature and the duration of anodization time. The vertical growth rate of the pores $(10-250 \mathrm{~nm} / \mathrm{min})$ was found to vary exponentially with anodizing voltage; however, it exhibits linear increment with the electrolyte temperature. The measured pore diameter (50-130 nm) shows a linear variation with anodizing voltage. The bottom barrier oxide layer was etched out by pore widening treatment to obtain through holes.
\end{abstract}

Keywords. Anodic aluminium oxide; barrier oxide layer; nano-pores.

\section{Introduction}

Since the successful growth of carbon nanotubes, great interest has been focused on one-dimensional materials, including nanotubes and nanowires, because of their unique structure and properties as well as potential applications in electronics, mechanics and optical devices (Morales and Lieber 1998; Li et al 1999). To actualize most of the proposed applications, it is quite important to obtain highly ordered nanostructure arrays. While there are technological as well as economical limitations in lithography for ultra large scale integrated fabrication in the forthcoming stage of sub-100 nm scale, much effort has been focused on nanostructure formation by self-organizing methods. Among them, anodic aluminium oxide (AAO) templates have received considerable attention in synthesizing nanostructure material due to their particular characteristics such as controllable pore diameter and periodicity, extremely narrow distribution of pore size, and the pore has ideal hexagonal shape (Masuda and Fukuda 1995; Masuda et al 1997; Jessensky et al 1998; Li et al 1998; Hwang et al 2002; Yuan et al 2004; Belwalkar et al 2008; Jeong et al 2009). This offers a promising route to synthesize a large-area, ordered nanostructure with high aspect ratio, which is quite difficult to form by conventional lithographic process.

The pore formation of AAO film was affected by several parameters, such as the anodic voltage, concentration and nature of acid solutions, temperature of electrolyte and the first anodization time. It is reported that sulphuric acid solution is suitable for smaller pores formation with diameter between 10 and $30 \mathrm{~nm}$ (Jessensky et al 1998; Li et al 2000;

*Author for correspondence (pchowdhury@css.nal.res.in)
Belwalkar et al 2008), whereas oxalic acid solution is used for medium size $(\sim 30-100 \mathrm{~nm})$ pore formations (Masuda and Fukuda 1995; Masuda et al 1997; Hwang et al 2002; Belwalkar et al 2008). In all of these studies, it was observed that the growth rate of the pore is highly correlated with the temperature and the anodic voltage. The aim of this paper is to study the effect of temperature and the anodic voltages for the fabrication of highly ordered pore arrays on aluminium thin films.

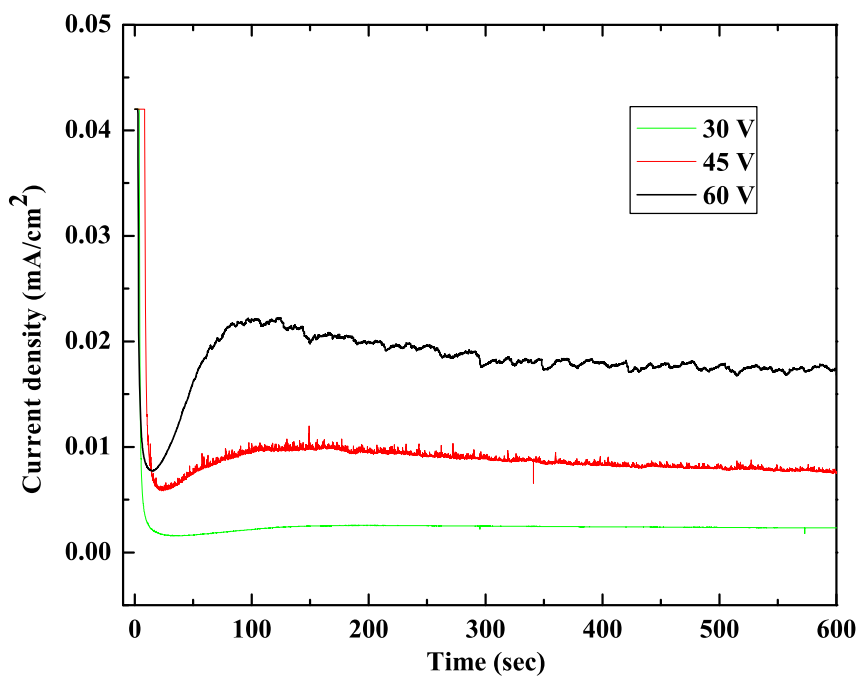

Figure 1. Current vs time $(I-t)$ characteristic curves for three different voltages of 30,45 and $60 \mathrm{~V}$ acquired at a temperature of $10^{\circ} \mathrm{C}$ 


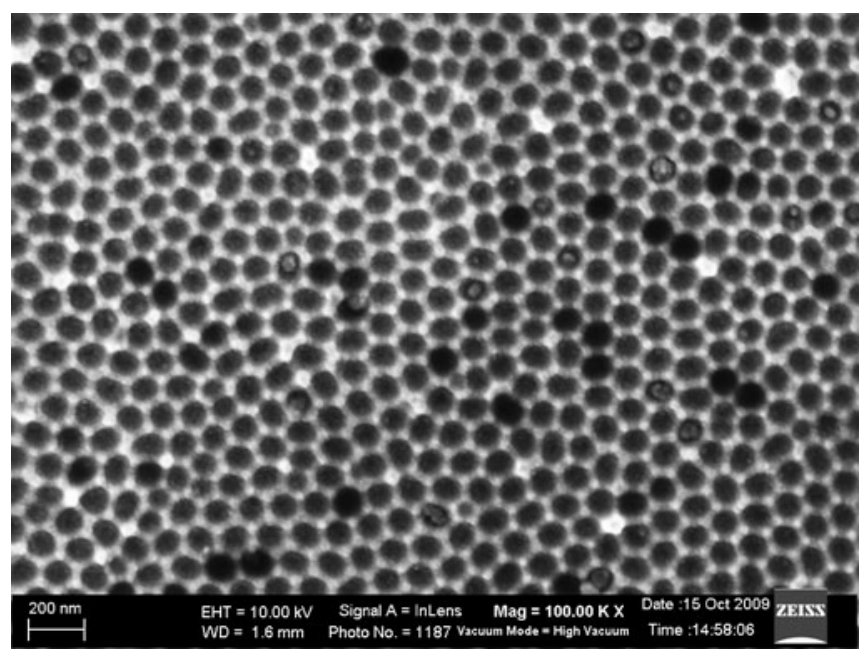

Figure 2. Top view of $\mathrm{Al}$ surface layer after removal of AAO film formed during 1 st anodization.

\section{Experimental}

A pure aluminium foil (purity 99.999\%) of thickness 130 $500 \mu \mathrm{m}$ was degreased in acetone by ultrasonication for $30 \mathrm{~min}$ to remove any adhering surface impurities. After ultrasonication, foil was rinsed with deionized water. A specimen of area $5 \times 1 \mathrm{~cm}^{2}$ was used as anode while Pt wire was used as a cathode. The distance between two electrodes was kept at $4 \mathrm{~cm}$. Two-step anodization was carried out in $0 \cdot 3 \mathrm{M}$ $\mathrm{C}_{2} \mathrm{H}_{2} \mathrm{O}_{4}$ solution (Masuda and Fukuda 1995; Hwang et al 2002). To obtain the optimum condition for ordered hexagonal pore structure, the electrolyte temperature, anodization voltage and duration of the anodization were varied. Temperature of the bath was kept at 5,10 and $15^{\circ} \mathrm{C}$, and applied anodizing voltages were 30,45 and $60 \mathrm{~V}$.

The first anodization was carried out for $5 \mathrm{~h}$ with constant temperature and anodizing voltage. After the 1st anodization, AAO layer was etched out in $6 \mathrm{wt} \%$ phosphoric acid and $1.8 \mathrm{wt} \%$ chromic acid at $60-80^{\circ} \mathrm{C}$ for $30-45 \mathrm{~min}$. The second anodization was carried out for $2 \mathrm{~h}$ under same condition
$30 \mathrm{~V}$

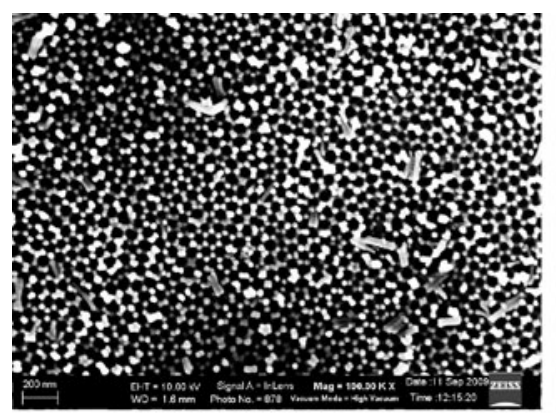

$10^{\circ} \mathrm{C}$

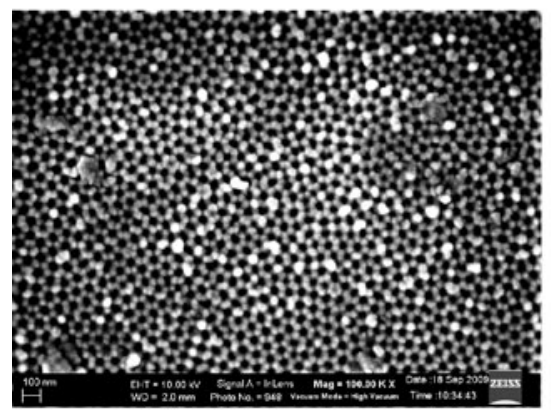

$15^{\circ} \mathrm{C}$

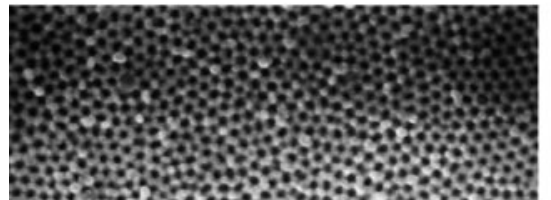

$45 \mathrm{~V}$
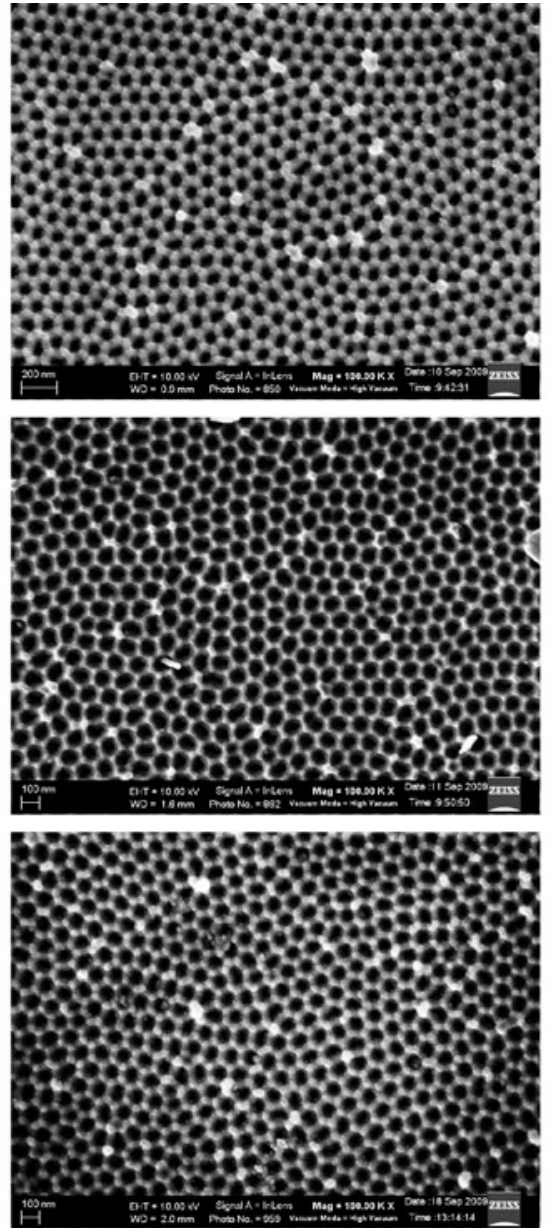

$60 \mathrm{~V}$
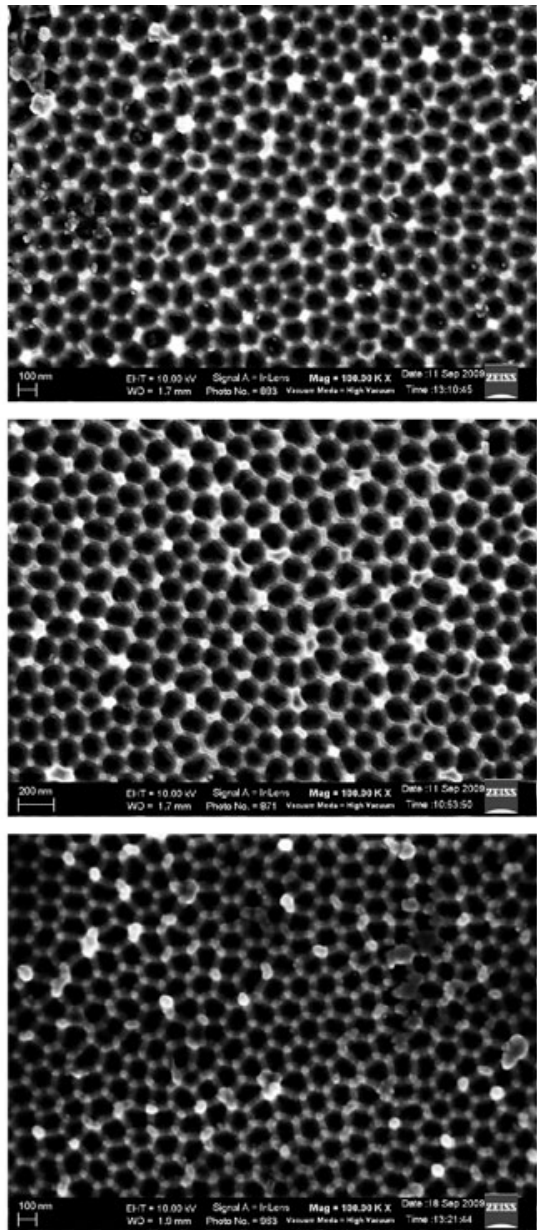

Figure 3. Top view of pore arrays grown at three different voltages and temperatures. 
as the first. The unreacted aluminium was removed by dipping the specimen in saturated solution of mercuric chloride $\left(\mathrm{HgCl}_{2}\right)$ for 10-30 min. The AAO template was then treated with $5 \mathrm{wt} \%$ phosphoric acid for 30-60 min to remove the bottom barrier oxide layer. The templates were again ultrasonicated in acetone. The structure of the AAO template was then investigated by field emission scanning electron microscopy (FESEM, Carl Zeiss).

\section{Results and discussion}

The mechanism behind the formation of pores is explained below:

$$
\begin{aligned}
& \text { Anode }: 2 \mathrm{Al}(s)+3 \mathrm{H}_{2} \mathrm{O} \rightarrow \mathrm{Al}_{2} \mathrm{O}_{3}(s)+6 \mathrm{H}^{+}(\mathrm{aq})+6 e^{-}, \\
& \text {Cathode }: 6 \mathrm{H}^{+}(1 \mathrm{M})+6 e^{-} \rightarrow 3 \mathrm{H}_{2}(g)
\end{aligned}
$$

Net reaction : $2 \mathrm{Al}(s)+3 \mathrm{H}_{2} \mathrm{O} \rightarrow \mathrm{Al}_{2} \mathrm{O}_{3}(s)+3 \mathrm{H}_{2}(g) \uparrow$.

The above reaction indicates that 6 electrons reduction are required to obtain 1 mole of $\mathrm{Al}_{2} \mathrm{O}_{3}$. However, experimentally it was observed that 10 electrons were transferred through the external circuit for the formation of 1 mole of $\mathrm{Al}_{2} \mathrm{O}_{3}(\mathrm{Wu}$ et al 2007). The additional electrons are due to the many side reactions. Among them oxygen evolution at the anode is the prominent one.

Figure 1 shows the current vs time $(I-t)$ curves measured at temperature, $10^{\circ} \mathrm{C}$ and for three different voltages 30,45 and $60 \mathrm{~V}$ at the beginning of first anodization. At the initial stage, surface oxidation of aluminium takes place for a period of 5-10 s, which is indicated by sharp decrease in the current density for all the applied voltages as shown in figure 1. Further continuation of anodization leads to rise in the current density (shown in figure 1). This is attributed to the fact that the localized dissolution of $\mathrm{Al}_{2} \mathrm{O}_{3}$ initiates at the oxide/electrolyte interface, which leads to the formation of vertical pores (Ma et al 2009). The indication of higher rate of increase of current density for higher voltages implies the higher dissolution rates in comparison to growth rate. After $200 \mathrm{~s}$ of anodization, the current density saturates to a constant value irrespective of the applied anodizing voltages as shown in figure 1. This reflects signature of equilibrium condition for growth and dissolution rates.

During the 1st anodization, hexagonal pores formed on the surface of $\mathrm{Al}$ foil are non-ordered due to the surface roughness. These non-ordered pores of AAO film should be removed by wet chemical etching using a mixture of phosphoric and chromic acids. Figure 2 shows the FESEM image of hexagonal pattern on the surface of aluminium specimen after etching off the AAO film. This highly ordered pattern after 1 st anodization of $5 \mathrm{~h}$ forms the seed for the growth of highly ordered pores during second anodization. Figure 3 shows the FESEM images of the top surfaces after second anodization at various voltages and temperatures. As shown in figure 3 , the best result was achieved at $45 \mathrm{~V}$ with
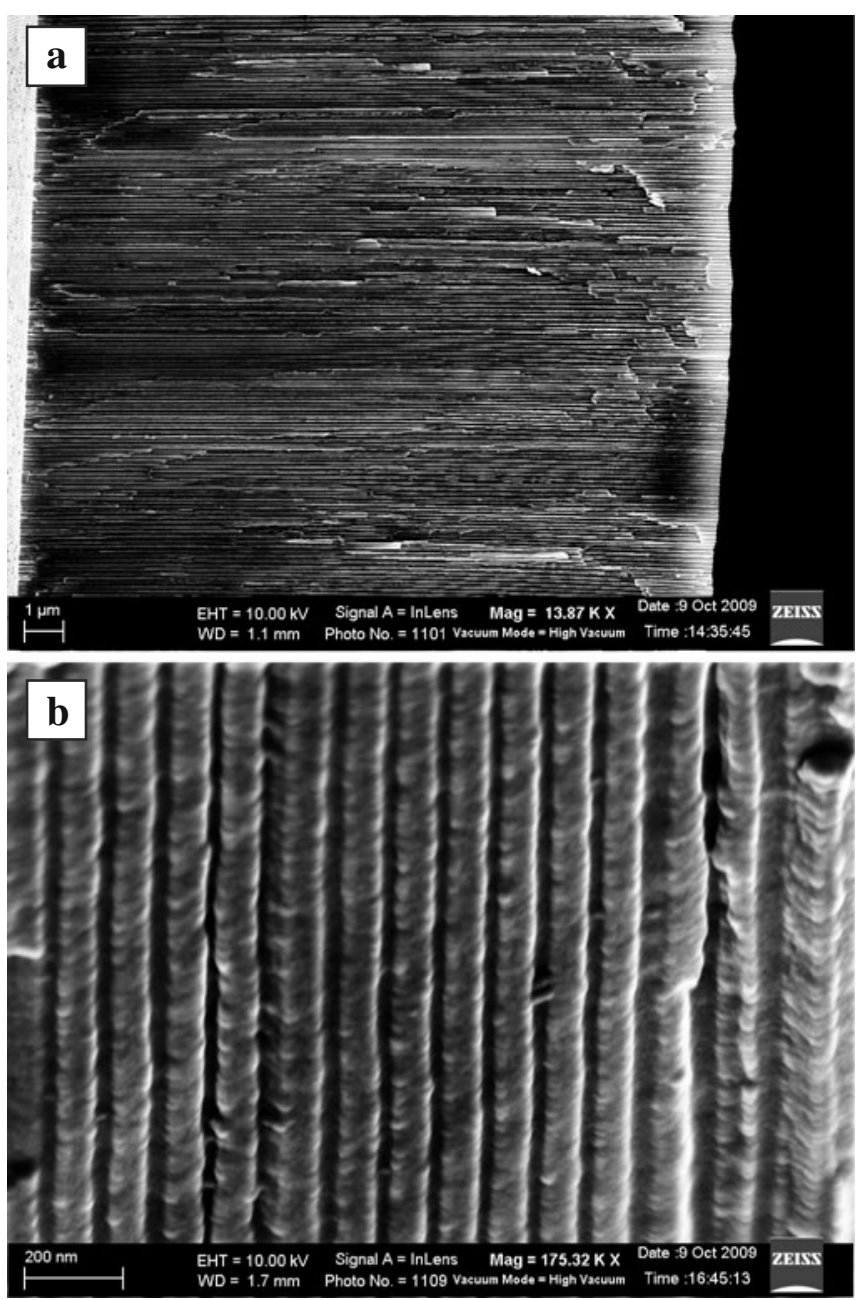

Figure 4. (a) Cross-section view of pore arrays grown at $10^{\circ} \mathrm{C}$ and $45 \mathrm{~V}$, and (b) same as (a) with higher magnification.

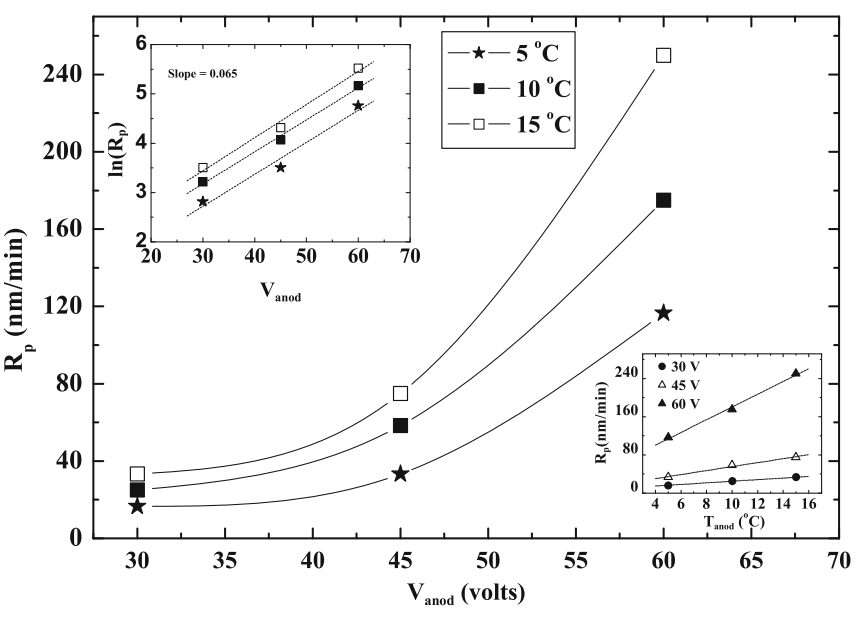

Figure 5. Growth rate as a function of anodizing voltage for three different temperatures. The upper inset shows the semi-logarithmic plot of growth rate as a function of anodizing voltage. The lower inset shows the linear variation of growth rate as a function of electrolyte temperature for three different anodizing voltages. 
an electrolyte temperature of $10^{\circ} \mathrm{C}$. The average pore diameter and the interpore distance were 85 and $133 \mathrm{~nm}$, respectively. The domain size with perfect order was $1-2 \mu \mathrm{m}$ and it was observed that it increases with increasing first anodization time. Similar observations were reported by several workers (Masuda and Fukuda 1995; Masuda et al 1997; Li et al 1998; Hwang et al 2002; Belwalkar et al 2008; Jeong et al 2009). At high voltages and high temperatures, the dissolution rate is higher due to enhanced current density. This causes an increase to the local temperature at the pore bottom which leads to non-uniform pore distribution due to local stresses and increased heat dissipation (Li et al 1998). At lower voltages and temperatures, though the pore formation is stable, however, the smaller interpore repulsive forces and low dissolution rates lead to lower volume expansion of oxide formation at the $\mathrm{Al} / \mathrm{Al}_{2} \mathrm{O}_{3}$ interface (Hwang et al 2002). This results in disordered pore structures at lower voltages and temperatures as shown in figure 3 . Figures 4(a) and (b) provide insight into the cross-sectional view of AAO template and the same with higher magnification, respectively.

Figure 5 presents the growth rate, $R_{\mathrm{p}}$, of AAO film as a function of anodizing voltage $\left(V_{\text {anod }}\right)$ for three different electrolyte temperatures, $T_{\text {anod }}$. The semi-logarithmic plots of $R_{\mathrm{p}}$ in upper inset of figure 4 show linear variation with $V_{\text {anod }}$, which indicates that $R_{\mathrm{p}}$ changes exponentially with $V_{\text {anod }}$ due to field enhanced dissolution irrespective of the temperature of the electrolyte. This contradicts with results of quadratic variation of $R_{\mathrm{p}}\left(V_{\text {anod }}\right)$ (Hwang et al 2002). However, as shown in lower inset of figure 5 , temperature-enhanced dissolution shows a linear pore growth rate for a constant value of $V_{\text {anod }}$.

Figure 6 shows the pore diameter, $D_{\mathrm{p}}$, as a function of $V_{\text {anod }}$ characteristics for three different temperatures 5, 10 and $15^{\circ} \mathrm{C}$ and it shows slight deviation from linearity at low

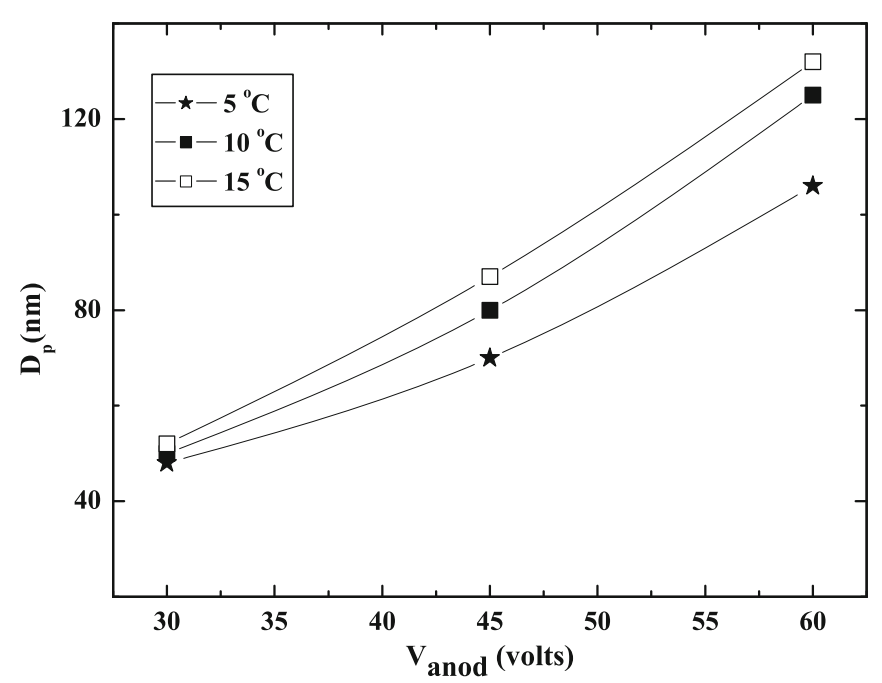

Figure 6. Variation of pore diameter obtained from FESEM images as a function of anodizing voltage.
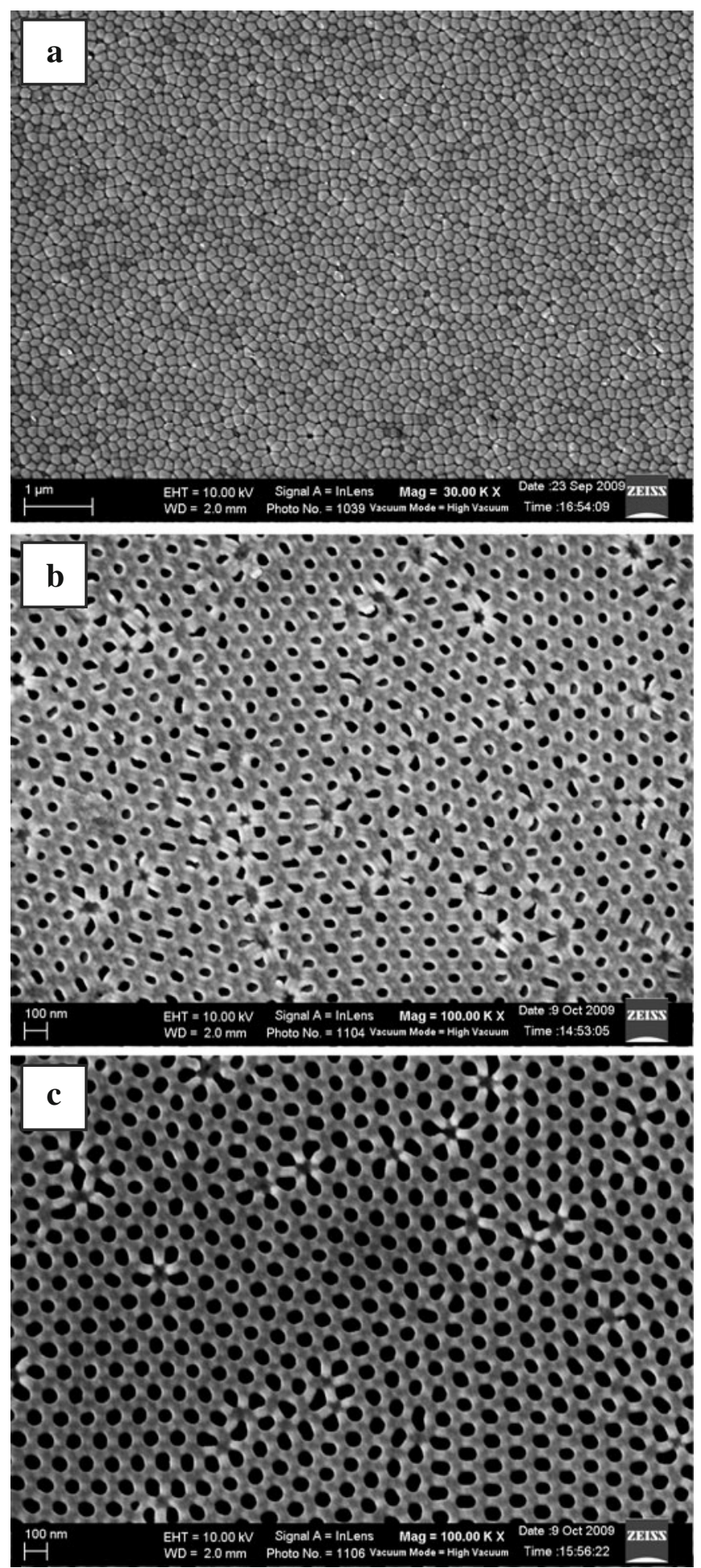

Figure 7. (a) Bottom barrier oxide layer of AAO template after treatment with $\mathrm{HgCl}_{2}$ solution. (b) and (c) FESEM images of bottom view of AAO template after 30 and 60 min of pore widening in phosphoric acid solution, respectively.

temperature. It also indicates that $D_{\mathrm{p}}$ has weak dependence on the electrolyte temperature and these results are similar to earlier reports (Li et al 2000; Hwang et al 2002). 
The enhancement of $D_{\mathrm{p}}$ with increase of $V_{\text {anod }}$ indicates the increase of localized charge accumulation at metal/oxide interface.

The process to vary the pore diameter without affecting pore density is called pore widening. This involves dissolution of AAO from pore walls and thinning of the barrier layer at the bottom in an acidic solution (Hwang et al 2002; Jeong et al 2009). Before the pore widening process, the specimen was immersed in saturated $\mathrm{HgCl}_{2}$ solution to etch out unreacted Al. Figure 7(a) shows the bottom view of AAO template with oxide barrier layer after treating with $\mathrm{HgCl}_{2}$. This shows the formation of barrier layer of thickness, $t_{\text {barrier }}$, which depends on the anodizing voltage and it is varied from 100-200 nm (Santos et al 2009). Removal of barrier layer is essential to use AAO template as a mask for synthesis of nanostructured materials by different deposition techniques. Figures 7(b) and (c) show the bottom view of AAO template after immersing in phosphoric acid solution for a duration of 30 and $60 \mathrm{~min}$, respectively. These micrographs clearly indicate complete removal of the barrier layer.

\section{Conclusions}

AAO templates were produced under various conditions of electrolyte temperatures and anodizing voltages. The vertical growth rate of pores was found to vary exponentially with anodizing voltage in comparison with linear variation with respect to the electrolyte temperature. Highly ordered hexagonal nano-pores of diameter $(\sim 80 \mathrm{~nm})$ with vertical growth rate of $60 \mathrm{~nm} / \mathrm{min}$ were obtained at $45 \mathrm{~V}, 0.3 \mathrm{M}$ oxalic acid and electrolyte temperature of $10^{\circ} \mathrm{C}$. Thinning of the bottom barrier layer was achieved by pore widening in phosphoric acid solution. The AAO templates with different pore diameters and thicknesses were successfully fabricated for the growth of nano-dimensional materials using both wet and dry deposition techniques.

\section{Acknowledgements}

The authors would like to thank the Director, National Aerospace Laboratories, Bangalore for giving support towards the development of nano-dimensional materials. $\mathrm{Mr}$ Siju is thanked for FESEM micrographs. The FESEM work was supported by CSIR Network Project (\# FAC-00-01-11).

\section{References}

Belwalkar A, Grasing E, Van Geertruyden W, Huang Z and Misiolek W Z 2008 J. Memb. Sci. 319192

Hwang S K, Jeong S H, Hwang H Y, Lee O J and Lee K H 2002 Korean J. Chem. Eng. 19467

Jeong S Y, An M C, Cho Y S, Kim D J, Paek M C and Kang K Y 2009 Curr. Appl. Phys. 9 S101

Jessensky O, Muller F and Gosele U 1998 J. Electrochem. Soc. 145 3735

Li A P, Muller F and Gosele U 2000 Electrochem. Solid-State. Lett. 3131

Li F, Zhang L, and Metzger R M 1998 Chem. Mater. 102470

Li J, Papadopoulos C and Xu J M 1999 Appl. Phys. Lett. 75367

Ma D, Li S and Liang C 2009 Corros. Sci. 51713

Masuda H and Fukuda K 1995 Science 2681466

Masuda H, Hasegwa F and Ono S 1997 J. Electrochem. Soc. 144 L127

Morales A M and Lieber C M 1998 Science 279208

Santos A, Vojkuvka L, Pallares J, Ferre-Borrull J and Marsal L F 2009 J. Electroanal. Chem. 632139

Wu Z, Richter C and Menon L 2007 J. Electrochem. Soc. 154 E8

Yuan H, He F Y, Sun D C and Xia X H 2004 Chem. Mater. 161841 